\title{
Joint Active Queue Management and Congestion Control Protocol for Healthcare Applications in Wireless Body Sensor Networks
}

\author{
Nazbanoo Farzaneh and Mohammad Hossein Yaghmaee \\ Ferdowsi University of Mashhad, Iran \\ Farzaneh@stu-mail.um.ac.ir, yaghmaee@ieee.org
}

\begin{abstract}
Wireless Body Sensor Network (WBSN) consists of a large number of distributed sensor nodes. Wireless sensor networks are offering the next evolution in biometrics and healthcare monitoring applications. The present paper proposes a congestion control protocol based on the learning automata which prevents the congestion by controlling the source rate. Furthermore, a new active queue management mechanism is developed. The main objective of the proposed active queue management mechanism is to control and manage the entry of each packet to sensor nodes based on learning automata. The proposed system is able to discriminate different physiological signals and assign them different priorities. Thus, it would be possible to provide better quality of service for transmitting highly important vital signs. The simulation results confirm that the proposed protocol improves system throughput and reduces delay and packet dropping.
\end{abstract}

Keywords: Active Queue Management; Congestion Control; Learning Automata; Transport protocol; Wireless Body Sensor Network.

\section{Introduction}

Wireless Body Sensor Networks (WBSNs) are a specific category of wireless sensor networks intended to operate in a pervasive manner for on-body applications [1]. The wireless body sensor network plays an important role for healthcare monitoring applications. For these applications, it is essential to be able to reliably collect physiological readings from humans via body sensor networks. Such networks could benefit from Quality of Service (QoS) mechanisms that support prioritized data streams, especially when the channel is impaired by interference or fading [2]. Congestion is an essential problem in wireless sensor networks. It not only wastes the scarce energy due to a large number of retransmissions and packet drops, but also hampers the event detection reliability.

This paper addresses the problem of congestion control in wireless body sensor networks. In this paper, a new queue management and adaptive source rate adjustment mechanism base on learning automata [3] for congestion control is proposed. The rest of this paper is organized as follows. In section 2 we present a review of related researches in transport protocols in WSN. The proposed protocol is explained In section 3. Section 4 evaluates proposed mechanism. We conclude the paper in section 5 . 


\section{Related Works}

The study of transport protocol in WSNs has been the subject of extensive research. In [4] a priority based rate and congestion control protocol for wireless multimedia sensor networks is presented. It consists of two major units. The Congestion Control Unit determines the congestion intensity by calculating the difference between the input and the output rate. The Service Differentiation Unit supports different QoS for different traffic classes. The RCRT [5] protocol uses the length of retransmission list as the indicator of congestion. The RCRT protocol uses its rate allocation component to assign rates to each flow in keeping with a rate allocation policy. RCRT boasts a NACK based end-to-end loss recovery scheme. In [6] a novel congestion control protocol for vital signs monitoring in wireless biomedical sensor networks is proposed. Based on the current congestion degree and the priority of its child nodes, the parent node dynamically computes and allocates the transmission rate for each of its children. LACAS [7] is Learning Automata-Based Congestion Avoidance Scheme for healthcare wireless sensor networks. In LACAS there is an automaton in every intermediate node which regulates the node's incoming rate for controlling congestion locally in that node. The automaton has five actions which are based on the rate with which an intermediate sensor node receives the packets from the source node. The rate of flow of data into a node for which there is the least number of packets dropped is considered to be the most optimal action.

\section{Proposed Model}

In WBSN sensors are attached to different patients. Each sensor is used to monitor a vital sign. Evidently, in such life-critical applications involving a large number of patients, congestion is extremely undesirable.

In this section we explain the proposed model in details. The proposed model consists of two different parts: 1) a learning and priority based AQM protocol and 2) a learning automata based transport protocol. For simplicity, we name the proposed model as LACCP (Learning Automata based Congestion Control Protocol). In the current study we consider two different classes, namely Critical and Normal. Critical class is dedicated to loss and delay intolerant traffics while Normal class is assigned to other traffics. In the following subsections, we describe these two parts in details.

\section{3-1 AQM Mechanism in Intermediate Nodes}

It is clear that when the packet arrival rate is more than departure rate, the node's queue will be filled. This, in turn, causes increased packet loss and delays. In a healthcare application, if a patient is known to have a special need, it would be possible to assign more priority to data transmitted from such a patient. In the proposed AQM protocol a packet is entered to the node's queue, based on its traffic class (Critical or Normal).

We use learning automata in order to adjust the packet arrival rate. In each intermediate node, there is a variable automaton which is shown by $\{\mathrm{A}, \mathrm{B}, \mathrm{P}, \mathrm{T}\}$ where $\mathrm{A}$ is a set four actions as follows: 
$\mathrm{A}=\{$ arrival_rate_incerase, no_change , arrival_rate_decrease_smoothly, arrival_rate_decrease_suddenly $\}. B$ includes the set of inputs and $P$ is the probability vector of the four automata actions. $P(n+1)=T[a(n), B(n), P(n)]$ is the learning automata where $n$ is the stage number. Table 1 gives a summarized definition of the four automata actions. Every automaton after selecting proper action receives feedback from the environment (in this paper the network). Based on the action, the transmission rate could be increased or decreased. The intermediate nodes' learning automata adjusts the transmission rate based on the number of packets in the queue and the number of lost packets. These two parameters provide a good assessment of the automata performance.

Table 1. Automaton actions in the intermediate nodes

\begin{tabular}{|c|c|}
\hline Action name & \multicolumn{1}{c|}{ Description } \\
\hline No change & $\begin{array}{c}\text { The network has reached stability and there is no need to change } \\
\text { the arrival rate. }\end{array}$ \\
\hline Arrival rate increase & $\begin{array}{c}\text { Since network feedback indicates small queue size and channel } \\
\text { load, the node can increase arrival rate in order to improve through- } \\
\text { put }\end{array}$ \\
\hline $\begin{array}{c}\text { Arrival rate decrease } \\
\text { smoothly }\end{array}$ & $\begin{array}{c}\text { Congestion is likely to occur or low density congestion has } \\
\text { occurred. Therefore, packet arrival rate is decreased smoothly in } \\
\text { order to avoid queue saturation. }\end{array}$ \\
\hline $\begin{array}{c}\text { Arrival rate decrease } \\
\text { suddenly }\end{array}$ & $\begin{array}{c}\text { Since congestion has occurred packet arrival rate is decreased } \\
\text { quickly in order to avoid packet loss and queue saturation. }\end{array}$ \\
\hline
\end{tabular}

Let $\Delta \mathrm{L}(\mathrm{t})$ and $\mathrm{L}(\mathrm{t})$ show the loss variation and loss rate in time $t$. The learning automata placed in each node calculates $\Delta \mathrm{L}(\mathrm{t})$ and $\Delta \mathrm{Q}(\mathrm{t})$ as follows:

$$
\begin{aligned}
& \Delta L(t)=L(t)-L(t-1), \\
& \Delta Q(t)=Q(t)-Q(t-1)
\end{aligned}
$$

Where $L(t-1)$ is the loss rate at time $t-1 . \Delta \mathrm{Q}(\mathrm{t})$ and $\mathrm{Q}(\mathrm{t})$ are queue length variation and queue length at time $t$, respectively. $\mathrm{Q}(\mathrm{t}-1)$ is the value of queue length at time $t$ 1. Network status can be determined based on the values of $\Delta \mathrm{L}$ and $\Delta \mathrm{Q}$. Different values of $\Delta \mathrm{L}$ and $\Delta \mathrm{Q}$ have different meanings and interpretations. For example when $\Delta \mathrm{L}<0$ or $\Delta \mathrm{L}>0$ this means that the packet loss rate has been decreased or increased, respectively. $\Delta \mathrm{L}$ defines the amount of decrease or increase in loss rate.

After choosing an action, the automata rewards or penalizes it based on network feedback as follows:

- If $(\Delta Q(t)<<0$ and $\Delta L(t)<=0)$ OR $(\Delta Q(t)>>0$ and $\Delta L(t)<0)$ the automaton is rewarded according to equation (2)

- If $(\Delta Q(t)>>0$ and $\Delta L(t)>0)$ OR $(\Delta Q(t)<<0$ and $\Delta L(t)>0$ and arrival_rate $<<$ initial_rate $)$ the automaton is penalized according to equation (3)

$$
\begin{aligned}
& P_{i}(n+1)=P_{i}(n)+a\left[1-P_{i}(n)\right] \\
& P_{j}(n+1)=(1-a) P_{j}(n) \quad \forall j j \neq i
\end{aligned}
$$




$$
\begin{aligned}
& P_{i}(n+1)=(1-b) P_{i}(n) \\
& P_{j}(n+1)=(b / r-1)+(1-a) P_{j}(n) \quad \forall j j \neq i
\end{aligned}
$$

In the above equations, " $\boldsymbol{a}$ " is the reward and " $\boldsymbol{b}$ " is the punishment parameter.

Shorter node's queue lengths lead to shorter packet waiting time for receiving service. Although decreasing packet arrival rate decreases queue length, it also increases the number of lost packets (packets which are lost due to low arrival rate) and decreases throughput. Therefore, the automaton has to strike a balance between queue length and network throughput. The number of lost packets will be increased if despite the changing of channel load, packet arrival rate remains constant. Therefore, an increase in packet loss indicates a lack of correspondence between arrival rate and the actual packet reception rate (channel load). Although the arrival rate should be decreased as queue length nears saturation, with the decrease of network throughput and queue length the arrival rate will be increased again. An increase in the number of lost packets causes the automata in the sink to decrease source transmission rate in order to decrease congestion and increase network throughput. Therefore, corporation between the automata placed in the intermediate nodes and the sink will decrease congestion.

Despite to LACAS the values of " $\boldsymbol{a}$ " and " $\boldsymbol{b}$ " in equations 2 and 3 are not constant and defined based on congestion level. Thus various congestion levels have different effect on automata. Although at the beginning of operation all probabilities $P_{i}$ are equal, as time passes the reward and punishment mechanism explained above will change these probabilities. Each node has two different arrival rates, namely Critical rate and Normal rate. The following relation is always true:

$$
\text { node arrival rate }=\text { Critical rate }+ \text { Normal rate }
$$

Thus if the number of received critical packets increase the number of normal arrival rate is decreased. The Critical and Normal rates are calculated as follows:

$$
\begin{aligned}
& \text { arrive }_{t}=w_{c} \text { arrive }_{c}+w_{n} \text { arrive }_{n} ; \quad w_{c} £ w_{n} \\
& \text { Prate }_{c}=w_{c} \text { arrive }_{c} / \text { arrive }_{t} \quad, \text { Prate }{ }_{n}=w_{n} \text { arrive }_{n} / \text { arrive }_{t} \\
& \text { Critical rate }=\operatorname{Prate}{ }_{c} * \text { rate, Normal rate }=\operatorname{Prate}{ }_{n}{ }^{*} \text { rate }
\end{aligned}
$$

where arrive $_{t}$, arrive $_{c}$ and arrive $_{n}$ are total received packets, total received Critical packets and total received Normal packets per time, respectively. $W_{c}$ and $w_{n}$ are the priority weights of Critical and Normal packets, respectively.

A weighted fair queue (WFQ) scheduler is used to schedule the incoming packets. To provide better quality of service for high priority traffic classes, the assigned weights used in the WFQ scheduler follows this rule: Critical Class has higher priority.

\subsection{Congestion Control in the Sink}

In addition to AQM mechanism which is placed in the intermediate nodes, in order to control and prevent congestion we need to adjust the source rate based on the congestion level in the network. To do so, we present a method based on the learning automaton in the sink node that learns about the network congestion status and assigns 
the proper rate to the source(patient) based on its current status(Critical or Normal). If this rate is too low network throughput decreases drastically. On the other hand, when source rate is too high it causes congestion and reduces network performance.

In the proposed protocol, variable learning automaton is used. This automaton has 3 actions as follows:

$A=\{$ source_rate_increase, no_change, source_rate_decrease $\}$. Table 2 gives a summarized definition of the three automaton actions.

Table 2. Definition of automaton actions in the sink

\begin{tabular}{|c|c|}
\hline Action name & Description \\
\hline No_change & $\begin{array}{c}\text { The network has reached stability and there is no need to } \\
\text { change the source rate. }\end{array}$ \\
\hline Source_rate_incerase & $\begin{array}{c}\text { Network congestion has been reduced; therefore the } \\
\text { source rate will be increased. }\end{array}$ \\
\hline Source_rate_decrease & $\begin{array}{c}\text { Due to congestion and increase in delay and packet loss, } \\
\text { the source rate should be decreased. }\end{array}$ \\
\hline
\end{tabular}

Choosing the proper action is based on the following learning parameters:1) delay,2) number of lost packets between two successful deliveries,3)throughput and 4)traffic class. To calculate the packet loss rate and delay variation, the learning automaton in the sink node uses the following equations:

$$
\begin{aligned}
\Delta L s(t) & =L s(t)-L s(t-1), \\
\Delta D(t) & =D(t)-D(t-1)
\end{aligned}
$$

where $\Delta \mathrm{Ls}(\mathrm{t})$ and $\mathrm{Ls}(\mathrm{t})$ denote the total loss rate variation and the value of packet loss at time $t . \operatorname{Ls}(\mathrm{t}-1)$ is the loss rate at time $t-1 . \Delta \mathrm{D}(\mathrm{t})$ and $\mathrm{D}(\mathrm{t})$ are delay variation and delay of received packet at time $t$, respectively. $\mathrm{D}(\mathrm{t}-1)$ is the value of delay in time $t$-1. In computing $L s(t)$ and $\mathrm{D}(\mathrm{t})$ Critical and Normal packets have different weights as follows:

$$
\begin{aligned}
& L s(t)=w_{c} L s_{c}(t)+w_{n} L s_{n}(t) \\
& D(t)=w_{c} D_{c}(t)+w_{n} D_{n}(t) \quad w_{c} \geq w_{n}
\end{aligned}
$$

Where $L s_{c}(t)$ and $L s_{n}(t)$ show the packet loss rate for Critical and Normal traffics at time $t$, respectively. $D_{c}(t)$ and $D_{n}(t)$ show the value of delay for Critical and Normal packet in time $t$. After choosing an action, the automata rewards or penalizes it based on network feedback as follows:

- If $(\Delta D(t)>0$ and $\Delta L(t)>0) \quad \mathrm{OR}(\Delta D(t)<0$ and $\Delta L(t)>0)$ the automaton is penalized according to equation $(2)$

- If $((\Delta D(t)<0$ and $\Delta L(t)<0))$ OR $(\Delta D(t)>0$ and $\Delta L(t)<0)$ the automaton is rewarded according to equation (3)

The larger the value of "number of lost packets" the higher the congestion will be. In addition, the delay of arrived packets at the sink indicates that the number of packets 
in the intermediate nodes' queue has been increased due to network congestion. Therefore, delay is an efficient parameter in determining network congestion.

By selecting an action, we expect to have a reduction in the number of lost packets and delay. If this does not realize, the automaton will penalize itself. Therefore, using the information obtained from the sink, the source finds out the optimal rate. This will lead to maximum throughput and congestion avoidance. The Critical patients get more bandwidth than others.

\section{Simulation Results}

To evaluate the performance of the proposed LACCP protocol, we simulated a wireless biomedical sensor network including 3 different patients. We used Opnet simulator [8]. In addition to the proposed protocol, the well known LACAS protocol was also implemented. The simulations were run using LAF protocol [9] as routing protocol and CSMA protocol as MAC layer protocol. The nodes were randomly distributed in the environment. At the beginning of the simulation, we assume all end sensor nodes (the patients) are in Normal condition. Patient 3 changed its status to Critical condition between time $\mathrm{t}=40 \mathrm{sec}$. and $\mathrm{t}=140 \mathrm{sec}$.

Table 3 shows the average end to end delay. It can be seen that the performance of LACCP protocol is better than LACAS protocol. Obviously, shorter queue lengths will cause shorter packet queuing delay. Critical packets are delay intolerant so these packets have a higher priority in entering and exiting the node, so they reach their destinations faster. Using a proper AQM mechanism which estimates the arrival rate based on queue length and channel load along with an automaton-based mechanism that determines source transmission rate will keep the queue length constant at a desirable size and avoid its sudden expansion.

Table 3 also shows the packet loss rate in the intermediate nodes. In sever congestion, the increase in channel load and queue length leads to a higher probability of loss in the intermediate nodes. So the number of accepted packets in the node is decreased. As seen in table3, the number of Critical lost packets is less than that of other classes. In LACAS only 5 rates are considered to be select by automaton which is not optimal. This indicates the adaptability of the proposed AQM mechanism in the intermediate nodes with the congestion control protocol in the sink.

Table 3. Impact of the service differentiation on average delay and packet loss

\begin{tabular}{|l||c||l||c|}
\hline \multicolumn{1}{|c||}{ Average delay } & Value & \multicolumn{1}{c||}{ Packet loss rate } & Value \\
\cline { 1 - 1 } Total for proposed protocol & 0.41 & Total for proposed protocol & 0.21 \\
\hline \hline For Critical Class & 0.24 & For Critical Class & 0.07 \\
\hline \hline For Normal Class & 0.36 & For Normal Class & 0.23 \\
\hline \hline Total for LACAS & 3.1 & Total for LACAS & 0.4 \\
\hline
\end{tabular}

Figure. 1 illustrates the remaining energy in the sensor nodes. The figure represents one of the network nodes in the bottleneck near the sink which is definitely the most energy consuming node. In the LACCP protocol, some messages are sent to the source from sink. So LACCP may use more energy than LACAS. It shows that there is no more difference between LACAS and LACCP in energy consumption. 
Figure 2 shows the number of total lost packets versus the simulation time. A comparison of the loss in the LACCP and LACAS protocols will show us that the LACCP protocol has a much lower level of loss than the LACAS protocol. This is because the learning automaton-based mechanism in the sink efficiently adjusts source transmission rate and avoids congestion and loss based on the feedback received from the network. Although the LACAS protocol adjusts the source transmission rate too, as the diagram shows the LACCP protocol is more successful in choosing the optimal source rate. Furthermore, in the LACCP, at time $\mathrm{t}=50 \mathrm{sec}$. the network becomes more compatible with the situation due to the learning of the automaton. Since source transmission rate is adjusted by the automaton, the number of lost packets is reduced and as the traffic increases lost packets remain almost constant.

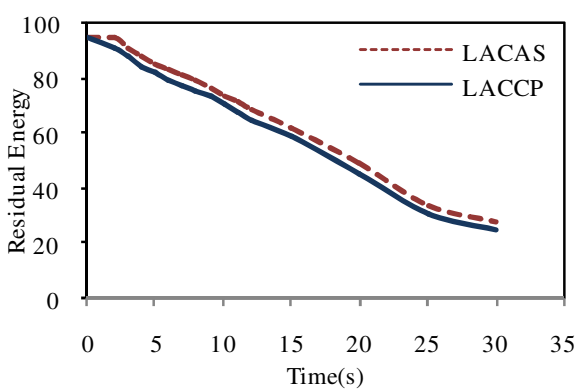

Fig. 1. Residual energy

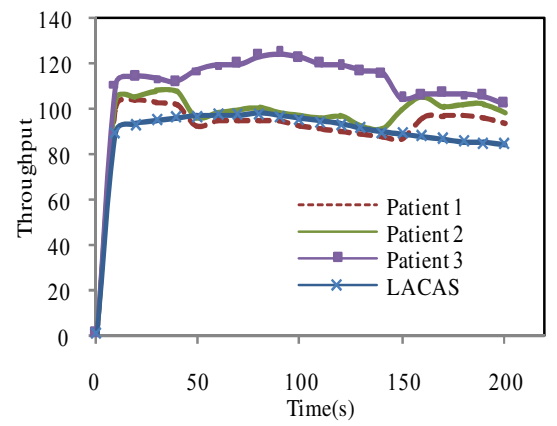

Fig. 3. Throughput versus time

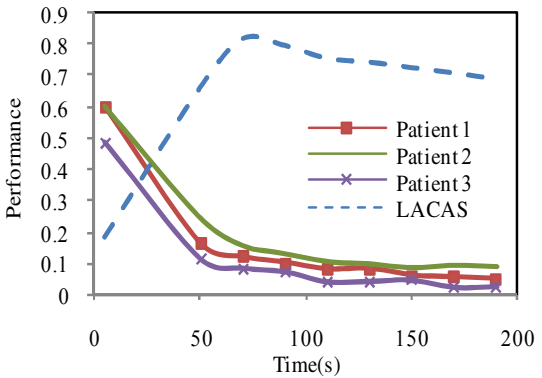

Fig. 2. Number of drop packets / number of packets generated by sensing nodes

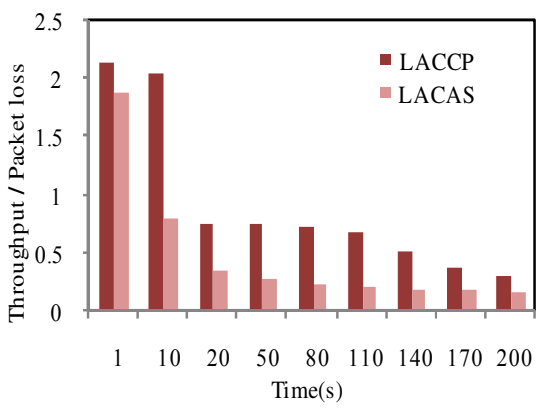

Fig. 4. eff parameter versus time

Figure 3 shows the throughput of both LACAS and LACCP protocols. We can observe that the proposed protocol can assign network bandwidth to each traffic class based on its priority. When patient 3 goes to Critical condition (during time interval [40s, 140s]), the system assigns more bandwidth to it. Since the LACAS protocol cannot discriminate between traffic classes, we have shown only its total throughput. Source reduces its rate due to packet loss avoidance. Reducing transmission rate 
should not lead to network throughput reduction. The following metric is defined in order to determine the impact of learning based congestion protocol (LACCP):

$$
\text { eff }=\frac{T}{L}
$$

Where $T$ is total throughput and $L$ is the average packet loss. The eff parameter shows the effectiveness of the protocol. The higher value of eff, the more favorite the answer. In figure 4 eff parameter is plotted versus time. LACCP protocol has higher throughput and lower packet loss than LACAS. So LACCP is more effective than LACAS.

\section{Conclusion}

In this paper we presented a congestion control protocol based on learning automaton for healthcare applications in WBSNs. The proposed congestion control protocol namely, LACCP can adjust intermediate node arrival rate and source rate using learning automata. Two different traffic classes namely Critical and Normal were considered. In order to control congestion, a mechanism based on the learning automaton has been placed in the sink. Using weighted scheduling mechanisms, higher priority classes are given better quality of service and more bandwidth than the lower priority classes. The simulation results indicate that the proposed protocol, by adjusting source rate, avoids loss caused by congestion. Furthermore, it has been shown that the LACCP achieve higher performance and lower packet loss than LACAS.

\section{References}

1. Ren, H.L., Meng, M.Q.H., Chen, X.J.: Physiological Information acquisition through wireless biomedical sensor networks. In: Proceeding of IEEE International Conference on Information Acquisition (July 2005)

2. Zhou, G., Liu, J., Wan, C., Yarvis, M., Stankovic, J.: BodyQoS: Adaptive and RadioAgnostic QoS for Body Sensor Networks, Infocom (April 2008)

3. Narendra, K.S., Thathachar, M.A.: Learning Automaton: An Introduction. Prentice Hall, Englewood Cliffs (1989)

4. Yaghmaee, M.H., Adjeroh, D.: Priority-based rate control for service differentiation and congestion control in wireless multimedia sensor networks. Computer Network Journal, 1798-1811 (2009)

5. Paek, J., Govindan, R.: RCRT: Rate-Controlled Reliable Transport for Wireless Sensor Networks. In: Proceedings of the ACM Conference on Embedded Networked Sensor Systems, Sensys (2007)

6. Yaghmaee, M.H., Adjeroh, D.: A Novel Congestion Control Protocol for Vital Signs Monitoring in Wireless Biomedical Sensor Networks, pp. 1-6, WCNC (2010)

7. Misra, S., Tiwari, V., Obaidat, M.S.: LACAS: Learning Automata-Based Congestion Avoidance Scheme for Healthcare Wireless Sensor Networks. IEEE Journal on Selected Areas in Communications 27(4), 466-479 (2009)

8. http://www. opnet.com/

9. Sabbineni, H., Chakrabarty, K.: Location-aided floooding: An energy efffcient data dissemination protocol for wireless sensor networks. IEEE Transactions on Computers 51(1), $36-46(2005)$ 